\title{
Photoacoustic effect as a liquid absorbance detector
}

\author{
Abdullah Atalar
}

\begin{abstract}
A theoretical treatment of the photoacoustic effect for a liquid enclosed in a cylindrical cell is given. Simple analytical expressions for the photoacoustic signal are derived for $\mathrm{cw}$ and pulse modulation schemes. Modulation frequency or pulse width dependence of the photoacoustic signal is discussed. For the cw modulation scheme it is found that more photoacoustic power is generated in a resonant cell than a nonresonant cell at the expense of bandwidth reduction. Conditions leading to $1 / f$ dependence of the signal on modulation frequency are given. Experimental results are presented that support the simplified treatments. Eventual sensitivities of various excitation schemes under optimum conditions are calculated.
\end{abstract}

\section{Introduction}

The photoacoustic effect that was discovered 100 years ago has recently been used to study the optical absorption of gases, ${ }^{1}$ liquids, ${ }^{2}$ and solids ${ }^{3}$ for modulation frequencies in the $10-\mathrm{Hz}$ to $1-\mathrm{GHz}$ range. It is an important alternative to standard techniques of measuring small absorption coefficients. Being a zero base line technique makes it particularly attractive.

The photoacoustic effect has been employed in absorbance measurements basically by two techniques. The first technique requires the sample to be placed in an enclosed cell. The incident light is chopped at frequencies ranging from $10 \mathrm{~Hz}$ to $1 \mathrm{kHz}$ (cw modulation), and a sensitive microphone is used to pick up the pressure variations. ${ }^{1}$ From a simple theory one may expect the photoacoustic signal to increase with the modulation frequency. ${ }^{4}$ However, the photoacoustic pressure signal for this geometry is observed to vary inversely with the chopping frequency. We will simplify the existing 5,6 theories as much as possible to provide a physical explanation for this behavior. The apparent discrepancy between these two expectations will be clarified, and it will be shown that at high frequencies the photoacoustic signal indeed increases with frequency. We will also discuss the effect of the light beam radius on the output signal.

The second technique uses pulsed light sources and piezoelectric transducers. Measurement of liquid ab-

When this work was done the author was with Hewlett-Packard Laboratories, Palo Alto, California 94304; he is now with Middle East Technical University, Department of Electrical Engineering, Inonu Bulvari, Ankara, Turkey.

Received 26 April 1980.

0003-6935/80/183204-07\$00.50/0.

(C) 1980 Optical Society of America. sorption coefficients as low as $10^{-6} \mathrm{~cm}^{-1}$ has been reported. ${ }^{7}$ A theoretical treatment of the pulsed system for spherical geometry was given. ${ }^{8,9}$ In this paper we will develop a model for a cylindrical geometry pulsed system and find an analytical expression for the thermoelastic pulse.

The results of the photoacoustic experiments using both modulation techniques (cw and pulsed) will be presented. The measurements will be compared to theoretical calculations. Reasonable agreement supports the validity of simplified treatments. Finally $\mathrm{cw}$ and pulsed modulation schemes will be compared, and the maximum achievable sensitivities for these systems will be given.

\section{Continuous Wave Modulation}

In this section we will consider the cw modulation technique for photoacoustic excitation. We will obtain expressions for the acoustic power for both free space and enclosed volume.

Referring to the cylindrical geometry shown in Fig. 1 , the liquid is illuminated by a collimated laser source of power $P_{0}$ and radius $r_{0}$. The laser light is sinusoidally modulated at frequency $\omega$. Intensity of the laser is represented by $I_{0} \exp \left(-r^{2} / r_{0}^{2}\right) \exp (-j \omega t)$. The liquid is contained in an infinitely long solid material of cylindrical shape.

The coupled thermoelastic equations for temperature $\tau$ and displacement potential $\Phi$ in a liquid are given by the following equations ${ }^{10}$ :

$$
\begin{aligned}
\kappa \nabla^{2} \tau-\rho c_{p}(\partial \tau / \partial t)+\beta T_{0} B \nabla^{2}(\partial \Phi / \partial t) & =-S_{t}, \\
\nabla^{2} \Phi-\left(1 / C^{2}\right)\left(\partial^{2} \Phi / \partial t^{2}\right) & =\beta \tau,
\end{aligned}
$$

where $\kappa$ is the thermal conductivity, $c_{p}$ is the heat capacity, $\rho$ is the density, $T_{0}$ is the ambient temperature, $B$ is the isothermal bulk modulus, $\beta$ is the volume 


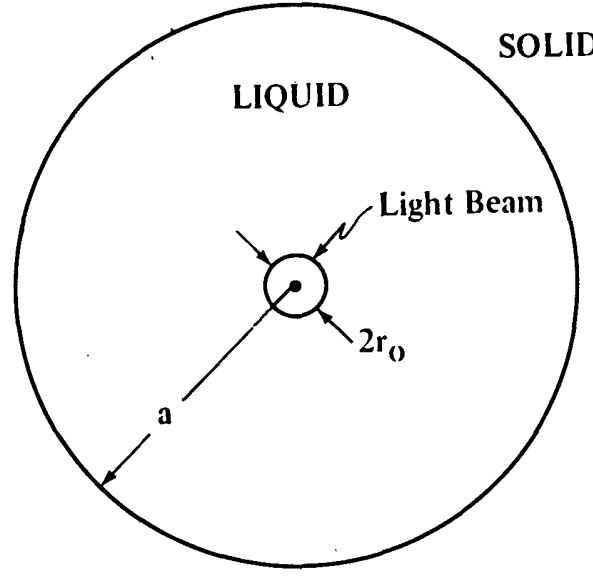

Fig. 1. Geometry of the cylindrical photoacoustic system under consideration.
This is an acoustic wave equation with a source term on the right-hand side. We will use the Green's function method to solve it.

\section{A. Free-Space Solution}

If the liquid is enclosed in an acoustically matched cell, there will be no internal reflections, and hence the solution in the cell is the free-space solution. In cylindrical coordinates the Green's function for $\nabla^{2} \Phi+k^{2} \Phi$ $=\delta\left(r-r^{\prime}\right) / r$ in free space is given by 8,11

$$
G\left(r, r^{\prime}\right)=-(j / 4) J_{0}\left(k r^{\prime}\right) H_{0}(d r) \text { for } r>r^{\prime} .
$$

Therefore, the solution of Eq. (4) is written as

$$
\Phi(r)=\frac{\pi \beta \alpha I_{0}}{2 \omega \rho c_{p}} H_{0}(k r) \int_{0}^{\infty} r^{\prime} J_{0}\left(k r^{\prime}\right) \exp \left(-r^{\prime 2} / r_{0}^{2}\right) d r^{\prime},
$$

where $k=\omega / C$. Since

$$
\int_{0}^{\infty} t \exp \left(-a^{2} t^{2}\right) J_{0}(b t) d t=\exp \left(-b^{2} / 4 a^{2}\right) / 2 a^{2}, 12
$$

we find

$$
\Phi(r)=\frac{\beta \alpha P_{0}}{4 \omega \rho c_{p}} \exp \left(-k^{2} r_{0}^{2} / 4\right) H_{0}(k r),
$$

where $P_{0}=I_{0} \pi r_{0}^{2}$ is the laser power, and $H_{0}$ is the zeroth-order Bessel function of the third kind (Hankel function). Radial velocity and stress are given by

$$
\begin{aligned}
v_{r} & =-j \omega \partial \Phi / \partial r=\left(j \beta \alpha P_{0} k\right) /\left(4 \rho c_{p}\right) \exp \left(-k^{2} r_{0}^{2} / 4\right) H_{1}(k r), \\
T_{r r} & =-\rho \omega^{2} \Phi=-\omega \beta \alpha P_{0} /\left(4 c_{p}\right) \exp \left(-k^{2} r_{0}^{2} / 4\right) H_{0}(k r) .
\end{aligned}
$$

Therefore, the acoustic power available in area $S$ at radial distance $r$ is ${ }^{13}$

$$
P_{A}=\operatorname{Re}\left(-v_{r}^{*} T_{r r}\right) S=\frac{\omega \beta^{2} \alpha^{2} P_{0}^{2}}{8 \pi \rho c_{p}^{2}} \exp \left(-k^{2} r_{0}^{2} / 2\right) \frac{S}{r} .
$$

The exponential factor in Eq. (6) expresses the dependence of the acoustic power on the light beam radius. The available power reduces if the beam radius exceeds the acoustic wavelength. For small beam size, Eq. (6) simplifies to

$$
P_{A}=\frac{\omega \beta^{2} \alpha^{2} P_{0}^{2}}{8 \pi \rho c_{p}^{2}} \frac{S}{r} .
$$

Equation (7) predicts that the acoustic power is proportional to the modulation frequency and to the square of the absorbed light power. This result is consistent with the results of Westervelt and Larson. ${ }^{4}$

\section{B. Enclosed Cell Solution}

If one uses a pressure transducer instead of a matched transducer, the free-space solution is not appropriate. In this case one has to include the effect of boundaries. We will now solve Eq. (4) when the liquid is contained in a cylindrical shaped solid container of radius $a$ and infinite in length. We will assume that the elastic constant and the density for the solid are large, so that we can use rigid boundary conditions. Furthermore, we will approximate spatial distribution of the laser beam with a delta function. We assume a solution of the following form:

$$
\nabla^{2} \Phi+\left(\omega^{2} / C^{2}\right) \Phi=j \beta \alpha I_{0} \exp \left(-r^{2} / r_{0}^{2}\right) /\left(\omega \rho c_{p}\right)
$$




$$
\Phi(r)=\frac{\beta \alpha P_{0}}{4 \omega \rho C_{p}}\left[H_{0}(k r)+A J_{0}(k r)\right] \text { for } r<a .
$$

The solution is selected in such a way that it reduces to the free-space solution as $a \rightarrow \infty$.

Now we apply the boundary condition at $r=a$; the displacement at $r=a$ is zero:

$$
u_{r}=\partial \phi /\left.\partial r\right|_{r=a}=0 \text { or } A=-H_{1}(k a) / J_{1}(k a),
$$

Hence displacement potential $\Phi$ is given by

$$
\Phi(r)=\frac{\beta \alpha P_{0}}{4 \omega \rho c_{p}}\left[H_{o}(k r)-\frac{H_{1}(k A)}{J_{1}(k a)} J_{0}(k r)\right] \text { for } r<a .
$$

The pressure at $r=a$ as experienced by a pressure transducer is

$$
p(a)=\frac{\omega \beta \alpha P_{0}}{4 c_{p}}\left[H_{0}(k a)-\frac{H_{1}(k a)}{J_{1}(k a)} J_{0}(k a)\right],
$$

or

$$
p(a)=\frac{\beta \alpha P_{0} C}{2 \pi c_{p} a} \frac{1}{J_{1}(k a)} .
$$

For small $k a, J_{1}(k a)$ behaves like $k a / 2$. Therefore, the pressure at $r=a$ is inversely proportional to modulation frequency. This $1 / f$ dependence agrees favorably with the experimental results reported in the literature. ${ }^{5}$

Equation (8) is plotted in Fig. 2 as a function of $k a$. The $1 / f$ dependence at low frequencies can be thought of as due to a resonance at zero frequency. At zero frequency the acoustic impedance of a liquid medium surrounded by a solid (even when it is nonrigid) is infinite. As the modulation frequency is increased the impedance of the liquid will decrease (the system goes off resonance), and hence the signal will decrease (it is a $1 / f$ dependence). This holds true for frequencies $\lesssim f$ $=c /(10 a)$. Above this frequency the pressure increases until the first resonance condition is satisfied. At the resonance frequency the impedance of the liquid is again infinite since it has rigid boundaries (which is an unreal condition). The same thing will occur at higher resonance frequencies that are at the zeros of the $J_{1}$ function.

Nonrigid boundary conditions can be taken into account if one includes the elastic constants and density of the surrounding medium. For an isotropic medium an analytical expression for the pressure can be found. If $C_{11}^{\prime}, C_{44}^{\prime}$ and $\rho^{\prime}$ are the elastic constants ${ }^{14}$ and density of the solid material, the pressure at $r=a$ is found to be

$p(a)=\frac{\omega \beta \alpha P_{0}}{4 c_{p}}\left\{H_{0}(k a)-\frac{\rho^{\prime} K a H_{1}(k a)\left[\frac{2 C_{44}^{\prime}}{C_{11}^{\prime}} \frac{H_{1}\left(k^{\prime} a\right)}{k^{\prime} a}-H_{0}\left(k^{\prime} a\right)\right]+\rho k^{\prime} a H_{0}(k a) H_{1}\left(k^{\prime} a\right)}{\rho^{\prime} k a J_{1}(k a)\left[\frac{2 C_{44}^{\prime}}{C_{11}^{\prime}} \frac{H_{1}\left(k^{\prime} a\right)}{k^{\prime} a}-H_{0}\left(k^{\prime} a\right)\right]+\rho k^{\prime} a J_{0}(k a) H_{1}\left(k^{\prime} a\right)} \cdot J_{0}(k a)\right\}$. medium. We observe that the pressure at the resonance frequencies is now finite. Moreover at low frequencies the pressure at the boundary is decreased as the acoustic mismatch between the liquid and solid is made smaller.

The power available in the solid medium can be found from the Poynting vector. We write the available power as $P_{A}=\operatorname{Re}\left(-v_{r}^{*} T_{r r}\right) S$, where $v_{r}$ is the particle velocity, and $T_{r r}$ is the stress at the boundary. This quantity is plotted in Fig. 3 for water as the liquid and PZT and Lucite as the surrounding medium. In the same graph

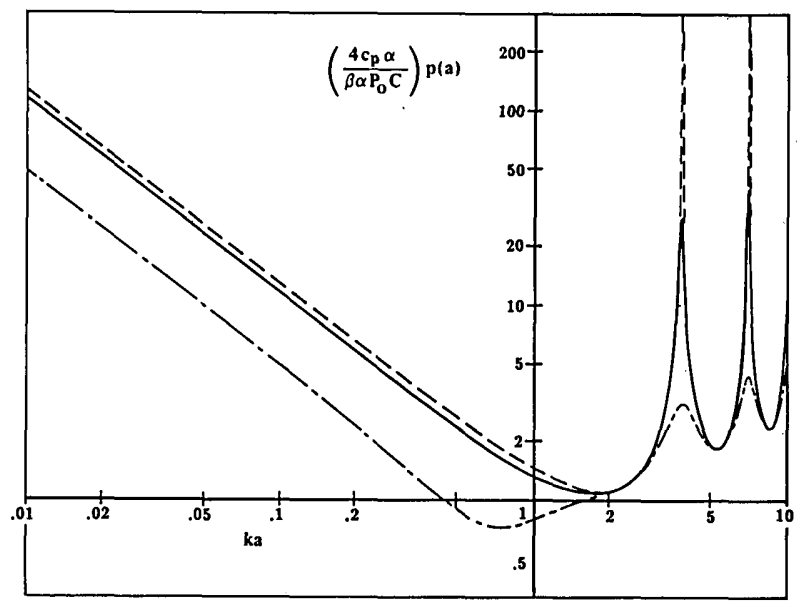

Fig. 2. Pressure at $r=a$ as a function of $k a$ for rigid boundary (dashed line), for water-Pyrex glass interface (solid line), and for water-Lucite interface (dot-dash line).

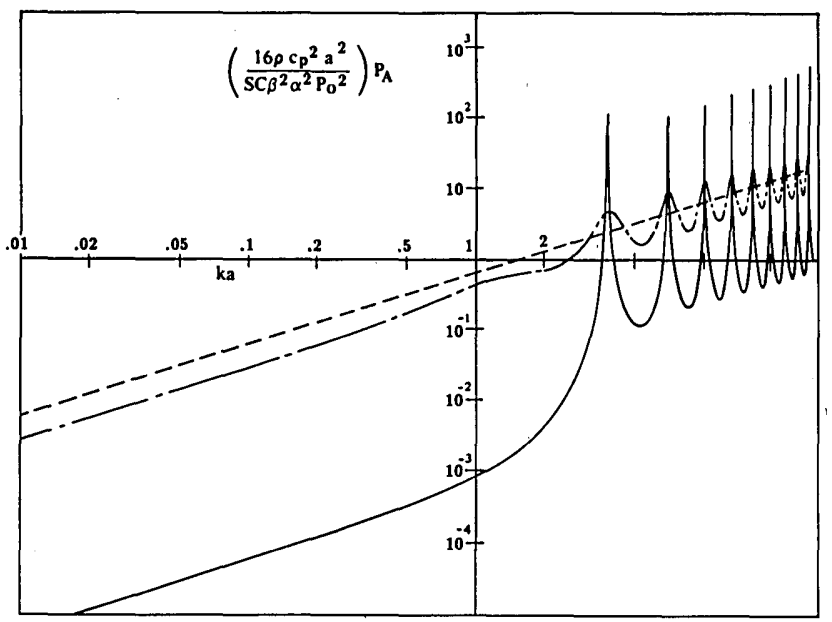

Fig. 3. Acoustic power available in the solid medium as a function of $k a$ for a matched cell (dashed line), for water-PZT cell (solid line), and for a water-Lucite cell (dot-dash line).

As a check we note that Eq. (9) reduces to Eq. (8) as $\rho^{\prime}$ $\rightarrow \infty$ (rigid boundary) and reduces to free-space solution for $\rho^{\prime}=\rho, C_{11}^{\prime}=C_{11}$, and $C_{44}^{\prime}=0$.

Equation (9) is also plotted in Fig. 2 for water as the liquid and Lucite and Pyrex glass as the surrounding 
the power available for a matched transducer is also shown [Eq. (7)]. Comparison of the free-space solution with the enclosed cell solution shows that a fully matched transducer that does not reflect any energy is not necessarily the optimum case, providing a resonant enclosed cell actually generates more acoustic power. This peculiar behavior can be explained by the nature of the source. The source can be seen as a displacement source (analogous to a current source in electrical circuits). Therefore, more power will be obtained if the impedance of the liquid as seen from the source can be increased. That is precisely what happens at the resonance: the source must work against the impedance of the solid rather than that of the liquid. One may expect that at the resonance the acoustic power will be increased by the ratio of impedances of solid and liquid. Therefore, we write the available acoustic power in the solid medium at the resonance as

$$
P_{A}^{r}=\frac{\omega_{r} \beta^{2} \alpha^{2} P_{0}^{2}}{8 \pi \rho c_{p}^{2}} \frac{S}{r} \frac{Z_{s}}{Z_{l}},
$$

where $\omega_{r}$ is the resonant frequency, $Z_{s}$ and $Z_{l}$ are the impedances of solid walls and liquid, respectively. Note that at the resonance there is a compromise between the output power and the usable bandwidth (i.e., data rate). Increasing the $Z_{s} / Z_{l}$ ratio results in a higher power but reduces the bandwidth. On the other hand, at antiresonant points the impedance seen by the source is reduced below the impedance of the liquid. Consequently, the available acoustic power is less than the free-space value.

\section{Pulsed Modulation}

We now solve the problem defined in Sec. II for a pulsed light source. We define the intensity of the light source as a function of space and time as $I_{0}$ $\exp \left(-r^{2} / r_{0}^{2}\right) f(t)$. Hence the energy of one pulse is given by

$$
E=2 \pi \iint r I_{0} \exp \left(-r^{2} / r_{0}^{2}\right) f(t) d r d t=I_{0} \pi r_{0}^{2} \int f(t) d t .
$$

Using the same reasoning of Sec. II, we neglect the thermal diffusion and find that

$$
\begin{aligned}
\frac{\partial \tau}{\partial t} & =\frac{\alpha I_{0}}{\rho c_{p}} \exp \left(-r^{2} / r_{0}^{2}\right) f(t), \\
\nabla^{2} \Phi-\frac{1}{C^{2}} \frac{\partial^{2} \Phi}{\partial t^{2}} & =\frac{\beta \alpha I_{0}}{\rho c_{p}} \exp \left(-r^{2} / r_{0}^{2}\right) g(t),
\end{aligned}
$$

where $g(t)=\int f(t) d t$.

To get rid of the time dependence we take the Laplace transform of both sides to find

$$
\nabla^{2} \Phi-\frac{s^{2}}{c^{2}} \Phi=\frac{\beta \alpha I_{0}}{\rho c_{p}} \exp \left(-r^{2} / r_{0}^{2}\right) \frac{\mathcal{L}[f(t)]}{s} .
$$

Using the same Green's function used in Sec. II with the identity of Eq. (5) we write

$$
\Phi(r, s)=-j \frac{\beta \alpha I_{0} \pi r_{0}^{2}}{4 \rho c_{p}} \frac{\mathcal{L}[f(t)]}{s} H_{0}(k r) \exp \left(\frac{s^{2} r_{0}^{2}}{4 C^{2}}\right),
$$

where $k=j s / C$.

We use the identity $K_{0}(z)=j(\pi / 2) H_{0}(j z)\left(K_{0}\right.$ is the modified Bessel function) and take the inverse Laplace transform of both sides to find

$$
\Phi(r, t)-\frac{\beta \alpha I_{0} r_{0}^{2}}{2 \rho c_{p}} \mathcal{L}^{-1}\left\{\frac{\mathcal{L}[f(t)]}{s} K_{0}\left(\frac{s}{C} r\right) \exp \frac{s^{2} r_{0}^{2}}{4 C^{2}}\right\} .
$$

If the radius of the laser beam is very small, the last item in the braces can be neglected. Furthermore we will assume that the light intensity changes as a triangular function of time. Let $f(t)$ be defined as

$$
f(t)=2 \frac{t}{T} u(t)-4 \frac{t-T / 2}{T} u\left(t-\frac{T}{2}\right)+2 \frac{t-T}{T} u(t-T),
$$

where $u(t)$ is the unit step function. $f(t)$ is plotted in Fig. 4(a).

Since the pressure is related to the displacement potential by $p(r, s)=-\rho s^{2} \Phi(r, s)$, we arrive at

$p(r, t) \cong$

$$
\frac{\beta \alpha I_{0} r_{0}^{2}}{2 c_{p}} \mathcal{L}^{-1}\left\{\frac{2}{T} \frac{K_{0}\left(\frac{s}{C} r\right)}{s}\left[1-2 \exp \left(-\frac{T}{2} s\right)+\exp (-T s)\right]\right\} .
$$

Using the transformation ${ }^{15}$

$$
\mathcal{L}^{-1}\left[\frac{k_{0}(b s)}{s}\right]=\left[\begin{array}{ll}
0 & t<b \\
\cosh ^{-1}\left(\frac{t}{b}\right) & t>b
\end{array}\right],
$$

we finally get

$$
\begin{aligned}
p(r, t)= & \frac{\beta \alpha I_{0} r_{0}^{2}}{T c_{p}}\left\{\cosh { }^{-1}\left(t \frac{Q}{r}\right) u\left(t-\frac{r}{C}\right)\right. \\
& -2 \cosh ^{-1}\left[\left(t-\frac{T}{2}\right) \frac{C}{r}\right] u\left(t-\frac{T}{2}-\frac{r}{C}\right) \\
& \left.+\cosh ^{-1}\left[(t-T) \frac{C}{r}\right] u\left(t-T-\frac{r}{C}\right)\right\} .
\end{aligned}
$$

This function is plotted in Fig. 4(b). Maximum pressure is reached at $t=T / 2+r / C$, and it is given by

$$
p_{\max }(r)=\frac{\beta \alpha I_{0} r_{0}^{2}}{T c_{p}} \cosh h^{-1}\left(1+\frac{C T}{2 r}\right) .
$$

Since the energy of one pulse is given by $E=$ $I_{0} \pi r_{0}^{2} T / 2$, we find

$$
p_{\max }(r)=\frac{2 \beta \alpha E}{T^{2} c_{p}} \cosh h^{-1}\left(1+\frac{C T}{2 r}\right)
$$

or approximately

$$
p_{\max }(r) \cong \frac{2 \beta \alpha E C^{1 / 2}}{T^{3 / 2} c_{p}} \frac{1}{\sqrt{r}} .
$$

The peak power available for a transducer of area $S$ at a distance $r$ is

$$
P_{A}(r)=\frac{2 \beta^{2} \alpha^{2} E^{2}}{T^{3} c_{p}^{2} \rho} \frac{S}{r} .
$$

We note that this result is very similar to Eq. (7) aside from a constant factor when we make the change of variable $T \rightarrow 2 \pi / \omega$ and $E / T \rightarrow P_{0}$. This is consistent with the previous results. For example, the linear dependence of $p_{\max }$ on thermal expansion coefficient $\beta$ has been experimentally verified. ${ }^{16}$ 


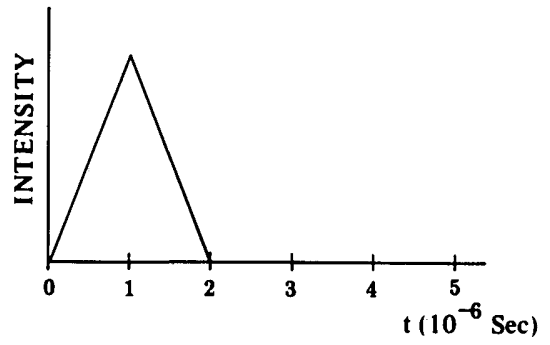

(a)

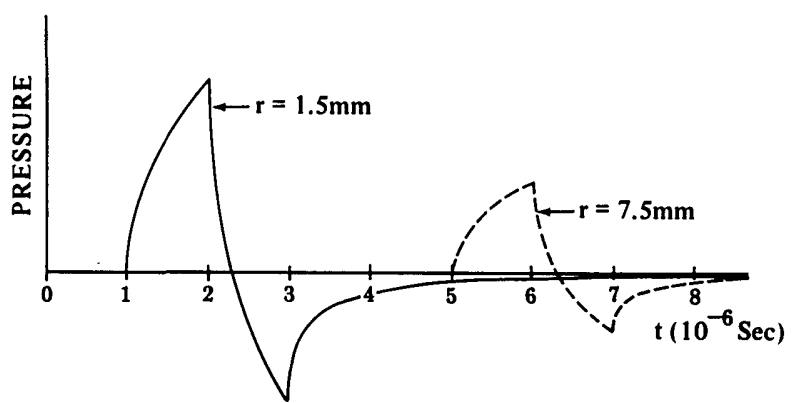

(b)

Fig. 4. (a) Intensity of the light beam as a function of time. (b) Photoacoustic pressure as a function of time at two different distances.

\section{Experimental Results}

In this section we will discuss the results of the photoacoustic experiments using $\mathrm{cw}$ and pulsed modulation and compare the findings to the theoretical equations presented in the previous sections. At low modulation frequencies nonresonant pressure transducers are available, and their sensitivities are expressed in terms of output voltage per unit pressure on the transducers. Therefore, we should use the pressure expression in Eq. (8) for a theoretical prediction. On the other hand, at high frequencies one can define the conversion loss of a transducer, and in this case the available acoustic power expression is useful.

\section{A. Continuous Wave Modulation Technique}

An Ar-ion laser capable of generating $1 \mathrm{~W}$ is used as the excitation source. The experiments have been carried out at low modulation frequencies $(10 \mathrm{~Hz})$ as well as at high modulation frequencies $(500 \mathrm{kHz})$.

At low frequencies the laser is modulated mechanically with a chopper wheel. The light is focused into a photoacoustic cell that consists of a cylindrical PZT material (Edo-Western Corp., Salt Lake City, Utah) with quartz windows on both sides. ${ }^{17}$ The output of the transducer (capacitance of $13 \mathrm{nF}$ ) is shunted with a $0.47-\mu \mathrm{F}$ capacitor to extend its low frequency range. The sensitivity of the transducer is estimated to be 4.3 $\mu \mathrm{V} / \mathrm{N} / \mathrm{m}^{2}{ }^{18}$ The output signal is measured with a lock-in amplifier for an aqueous solution with absorption coefficient $\alpha=0.027 / \mathrm{cm}$ (measured with a spectrophotometer). The results are shown in Fig. 5 as a function of modulation frequency. The theoretical results are found using Eq. (8) with $\beta=1.84 \times 10^{-4} /{ }^{\circ} \mathrm{C}$, $P_{0}=0.33 \mathrm{~W}, c_{p}=4178 \mathrm{~J} / \mathrm{kg}{ }^{\circ} \mathrm{C}$, and $a=1.6 \mathrm{~cm}$. We get
$p(a)\left(\mathrm{N} / \mathrm{m}^{2}\right)=17.5 / f(\mathrm{~Hz})$. Applying the sensitivity of the transducer we arrive at $V(\mu \mathrm{V})=75 / f(\mathrm{~Hz})$. This is also plotted in Fig. 5 as a solid line.

At high modulation frequencies $(\sim 500 \mathrm{kHz})$ an acoustooptic modulator is used. The modulated light is focused into a photoacoustic cell that has aluminum walls and quartz windows. A cylindrically focused immersion transducer (KB-Aerotech) converts the acoustic energy into the electrical energy with $9.5-\mathrm{dB}$ loss. The transducer's output is preamplified and then detected with a lock-in amplifier. The experiment is carried out with an aqueous solution of absorption coefficient $\alpha=0.12 / \mathrm{cm}$. The output signal is observed to change dramatically with slight changes in the modulation frequency. This behavior agrees with our expectations. The modulation frequency is fine tuned for maximum signal. Under this condition the output signal is plotted in Fig. 5 as a function of input laser power. A theoretical value can be found using Eq. (10), which gives the available power at the resonance frequencies. With $\omega_{r}=2 \pi 500 \times 10^{3}, S=5 \mathrm{~cm}^{2}, r=2.5$ $\mathrm{cm}$, and $Z_{s} / Z_{l}=11.5$, we find $P_{A}^{r}=8.14 \times 10^{-12} P_{0}^{2}$.

Taking the conversion loss of the transducer into account, the voltage output of the transducer should be $V=6.76 \times 10^{-6} P_{0} \mathrm{~V}$. This is plotted in Fig. 6 as a solid line. The expected signal is less than the measured signal, because our theory assumes that the solid walls extend to infinity. The finite thickness of the walls causes additional reflections and increases the output signal. Note that, if $1 / f$ dependence had been true for all frequencies, we should have obtained a signal in the nanovolt range at this frequency. Our signal is 3 orders of magnitude greater, supporting the theory that $1 / f$ dependence holds true only for low frequencies.

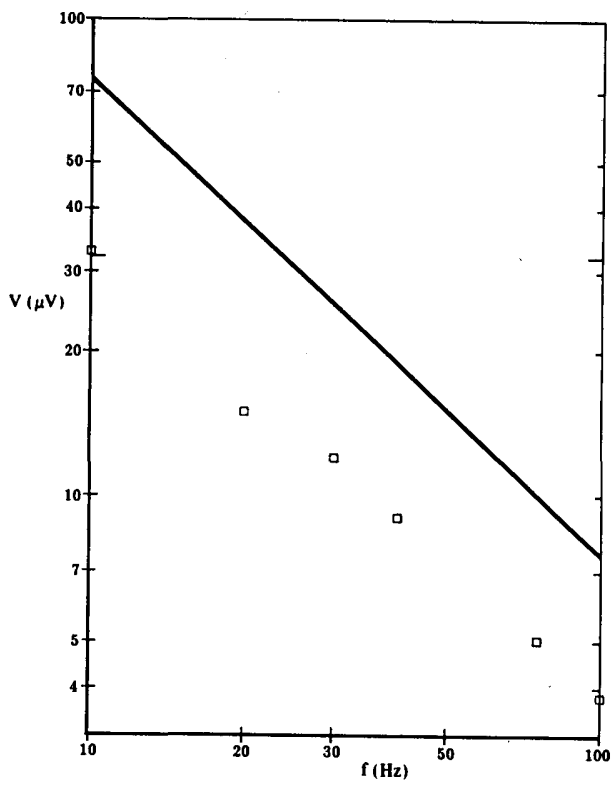

Fig. 5. Measured (squares) and calculated (solid line) photoacoustic voltage as a function of modulation frequency. Disagreement between the measured and theoretical values may be attributed to an overestimation of sensitivity of transducer and to neglecting the energy lost due to fluorescence. 


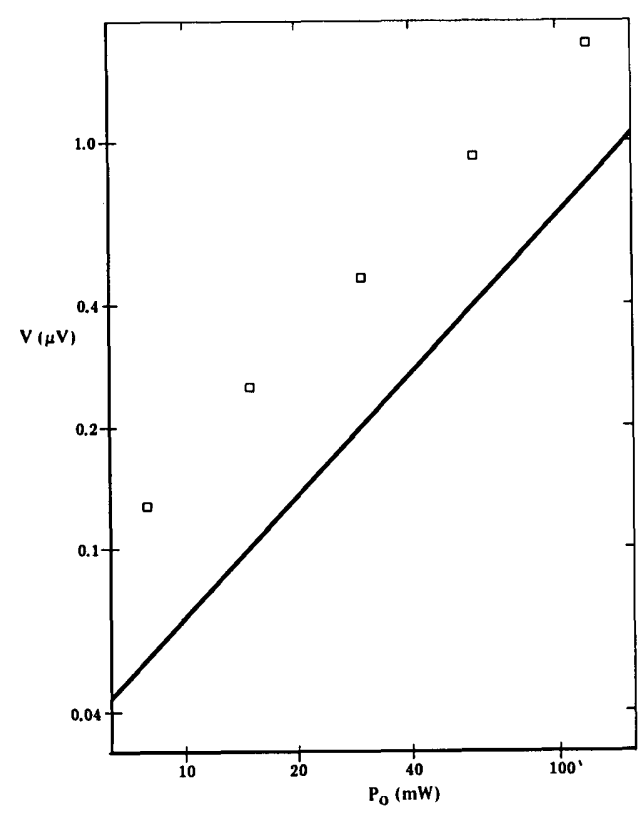

Fig. 6. Measured (squares) and calculated (solid line) photoacoustic voltage at $500 \mathrm{kHz}$ as a function of light power. Theoretical values are less than the measured values, since the finite thickness of walls causes additional reflections that are neglected in theory.

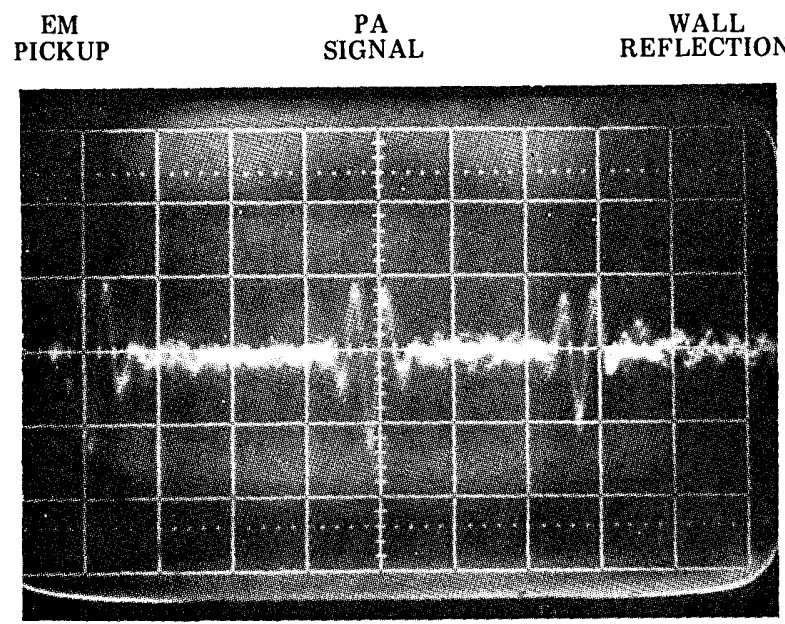

$5 \mu \mathrm{Sec} / \mathrm{Div}$

Fig. 7. Oscilloscope photo of the transducer output in response to a short light pulse illuminating the photoacoustic cell.

\section{B. Pulse Modulation Technique}

In this experiment we used an EG\&G flashlamp (FX-279U) as the photoacoustic exciter. The light source has a spatial extent of $1.5 \mathrm{~mm}$, and it has an UV rich spectrum. A short pulse of $1-\mu$ sec duration is obtained by discharging to the flashlamp $320 \mathrm{~mJ}$ stored on a capacitor. Due to the isotropic radiation only a small fraction of the generated light could be employed. The usable light energy within the first $2 \mu \mathrm{sec}$ is measured to be $126 \mu \mathrm{J}$. The transducer was the same as that used in the cw modulation experiment. We used 1:10000 acetone in water as the absorbing medium. The absorption spectra of the solution is measured to be $0.025 / \mathrm{cm}$ in the $240-290-\mathrm{nm}$ range. Comparison of this range with the spectrum of the flashlamp shows that the usable light energy is only $21 \mu \mathrm{J}$. Substituting these values in Eq. (12) with $r=3.8 \mathrm{~cm}$ gives $P_{A}=2.14 \times$ $10^{-11} \mathrm{~W}$. Conversion loss and finite bandwidth of the transducer result in a $20-\mathrm{dB}$ loss, giving an output voltage of $3.3 \mu \mathrm{V}$.

In Fig. 7 an oscilloscope photo of the amplifier output is shown. The first pulse corresponds to electromagnetic (EM) pickup, the second pulse is the photoacoustic signal, and the last one is the acoustic reflection from the bottom of the cell. The photoacoustic signal has $1.5-\mu \mathrm{V}$ amplitude, which is within $7 \mathrm{~dB}$ of the expected value. The shapes of all the pulses are determined by the bandpass network formed by the transducer and its matching network. With a bandwidth of $200 \mathrm{kHz}$, the impulse response of the transducer lasts $5 \mu \mathrm{sec}$, which is short enough to separate the EM pickup and wall reflections but not short enough to be compared to the theoretical pulse shape.

\section{v. Discussion}

In this section we will compare the various techniques used in photoacoustic absorbance detection and calculate the eventual sensitivities for these techniques.

Equations (7) and (12), which express the available acoustic power for $\mathrm{cw}$ and pulsed excitations, are repeated for comparison:

$$
\begin{aligned}
& P_{A}=\frac{\omega}{8 \pi} \frac{\beta^{2} \alpha^{2}}{\rho c_{p}^{2}} P_{0}^{2} \frac{S}{r}, \\
& P_{A}=\frac{2}{T} \frac{\beta^{2} \alpha^{2}}{\rho c_{p}^{2}}\left(\frac{E}{T}\right)^{2} \frac{S}{r} .
\end{aligned}
$$

For cw modulation the photoacoustic signal power is proportional to the modulation frequency. That is, to measure an absorption coefficient that is half as small, one has to quadruple the modulation frequency. One runs into difficulty when the size of the laser beam becomes comparable to the acoustic wavelength. This is due to the exponential factor in Eq. (6). Assuming a minimum laser beam radius of $40 \mu \mathrm{m}$, the maximum modulation frequency in water is $12 \mathrm{MHz}$. Making a resonant cell increases the output power by the impedance ratio as shown in Eq. (10), but this is at the expense of reduced bandwidth.

For the pulse modulation case the signal amplitude is proportional to the energy of the pulse. We have to note that the thermal noise level for a pulsed system is greater than that for a cw system of the same output bandwidth by a factor equal to the reciprocal of the duty cycle. This statement assumes that a boxcar integrator is used in the pulsed system. Equation (12) tells us that, if pulse duration $T$ is shortened to $0.63 T$, the output power will be doubled. However, due to the reduction of the duty cycle, noise increases by $2 \mathrm{~dB}$. Hence the actual gain in SNR is $4 \mathrm{~dB}$. Therefore, to increase the SNR by $6 \mathrm{~dB}$ one has to cut the pulse size in half without changing the pulse energy.

For cw modulation at low frequencies, one has to use nonresonant transducers. In this case Eq. (7) is not useful, instead we employ Eq. (8) to find the output voltage of the transducer: 
Table I. Minimum Detectable Absorption Coefflcients For Various Techniques Under the Conditions Given in the Text

\begin{tabular}{lccc}
\hline & $\begin{array}{c}\text { Continuous } \\
\text { wave } \\
\text { modulation }\end{array}$ & $\begin{array}{c}\text { Pulse } \\
\text { modulation }\end{array}$ & $\begin{array}{c}\text { Continuous } \\
\text { wave } \\
\text { modulation }\end{array}$ \\
\hline$\alpha_{\min }\left(\mathrm{cm}^{-1}\right)$ & $f=1 \mathrm{MHz}$ & $T=1 \mu \mathrm{sec}$ & $f=10 \mathrm{~Hz}$ \\
$\mathrm{H}_{2} \mathrm{O}$ solvent & $3.69 \times 10^{-6 /}$ & $1.3 \times 10^{-9} / E(\mathrm{~J})$ & $\begin{array}{c}2.43 \times \\
10^{-6} /\end{array}$ \\
& $P_{0}(\mathrm{~W})$ & & $P_{0}(\mathrm{~W})$ \\
$\mathrm{CCl}_{4}$ solvent & $1.42 \times 10^{-7} /$ & $4.98 \times 10^{-11 / E(J)}$ & $9.34 \times$ \\
& $P_{0}(\mathrm{~W})$ & & $10^{-8 /}$ \\
& & & $P_{0}(\mathrm{~W})$ \\
\hline
\end{tabular}

$$
V_{p}=K \frac{\beta \alpha P_{0} C^{2}}{2 \pi c_{p} a^{2}}(1 / \omega)
$$

where $K$ is the sensitivity of the transducer in $\mathrm{V} / \mathrm{N} / \mathrm{m}^{2}$. The output voltage is inversely proportional to the modulation frequency and square of the cell radius. The other factors are similar to Eq. (7) or (12). One can use a radially polarized piezoelectric tube as the transducer. For optimum results a high piezoelectric constant material must be used. The transducer can be modeled as a voltage source in series with a capacitor. The minimum detectable voltage under unity SNR for a capacitive source driving a resistive load is given by $\sqrt{4 k T B / R} / \omega C$, where $C$ is the source capacitance, $R$ is the load resistance, and $B$ is the bandwidth. Sensitivity of a cylindrical transducer depends on the radius rather than the thickness of the piezoelectric tube. ${ }^{19}$ Therefore, the piezoelectric tube should be as thin as possible to increase the capacitance. But if the tube is too thin, the rigid boundary condition will no longer be true, and the pressure will decrease.

For purposes of comparison, we will calculate the minimum detectable absorption coefficient for all three techniques as a function of light input power or energy. We take a $1-\mathrm{cm}$ long cell enclosed by a cylindrical transducer. The assumptions for different techniques are given below:

(1) Continuous wave modulation (high frequency): modulation frequency is $1 \mathrm{MHz}$, the cell is at resonance with a wall-to-liquid impedance ratio of 20 , one-way conversion loss of the transducer is $10 \mathrm{~dB}$.

(2) Continuous wave modulation (low frequency): the cell radius is $1 \mathrm{~cm}$, modulation frequency is $10 \mathrm{~Hz}$, the transducer capacitance is $10 \mathrm{nF}$ (with a load resistance of $10 \mathrm{M} \Omega$ ), the sensitivity is $150 \mu \mathrm{V} / \mathrm{N} / \mathrm{m}^{2}$.

(3) Pulse modulation: pulse duration $1 \mu \mathrm{sec}$, repetition rate is $20 \mathrm{~Hz}$, one-way conversion loss of the transducer is $10 \mathrm{~dB}$.

For all systems the output bandwidth is $1 \mathrm{~Hz}$, and the noise figure of the preamplifier is $3 \mathrm{~dB}$. The minimum absorption coefficient is defined when the SNR is unity. The thermal noise is assumed to be the only noise source. The results are shown in Table I.

To achieve the sensitivities shown for cw modulation techniques, one has to solve the spurious signal problem. Absorption in the windows, light scattering, and electromagnetic pickup will cause these spurious signals. The pulse system is preferable over cw excitation if these signals are unavoidable. Moreover, for the same sensitivity the average input light power is less in pulsed modulation than it is in cw modulation. In other words, for the same average power the pulse system is more sensitive than the cw system by a factor inversely proportional to the duty cycle. This arises from the fact that the photoacoustic effect is a nonlinear process as far as input and output powers are concerned. Using high pulse energies one should be able to measure absorption coefficients not obtainable by other techniques.

\section{Conclusion}

We have shown that $1 / f$ frequency dependence at low frequencies is observed only with pressure transducers in enclosed cells. Actually the generated acoustic power increases as the modulation frequency is increased in the $\mathrm{cw}$ system or as the pulse duration is decreased in the pulse system. We found that providing a matched cell in the cw case is not the optimum solution. At the expense of bandwidth reduction a resonant cell would generate more power than a nonresonant cell. The pulsed excitation scheme is preferable over the cw system since it solves the spurious signal problem, and it requires less average light power for the same sensitivity. Even though the photoacoustic conversion is not an efficient mechanism, use of high pulse energies would make the measurement of very low absorption coefficients possible, since it is a zero base line technique.

The author would like to acknowledge Dave Bloom for guidance and valuable discussions during the course of the work and critical reading of the manuscript. $\mathrm{He}$ would like to thank Bob Board for encouragement and Becky Tamura for preparation of the manuscript.

\section{References}

1. L. B. Kreuzer, Anal. Chem. 46, 235A (1974).

2. A. C. Tam, C. K. N. Patel, and R. J. Kerl, Opt. Lett. 4, 81 (1979).

3. M. M. Farrow, R. K. Burnham, M. Auzanneau, S. L. Olsen, N. Purdie, and M. E. Eyring, Appl. Opt. 17, 1093 (1978).

4. P. J. Westervelt and R. S. Larson, J. Acoust. Soc. Am. 54, 121 (1973).

5. A. Rosencwaig and A. Gersho, J. Appl. Phys. 47, 64 (1976).

6. F. A. McDonald and G. C. Wetsel, Jr., J. Appl. Phys. 49, 2313 (1978).

7. C. K. N. Patel and A. C. Tam, Appl. Phys. Lett. 34, 467 (1979).

8. C. L. Hu, J. Acoust. Soc. Am. 46, 728 (1969).

9. M. W. Sigrist and F. K. Kneubuhl, J. Acoust. Soc. Am. 64, 1652 (1978).

10. F. A. McDonald, Appl. Opt. 18, 1363 (1979).

11. G. Tyras, Radiation and Propagation of Electromagnetic Waves (Academic, New York, 1969), p. 486.

12. M. Abramowitz and I. A. Stegun, Eds., Handbook of Mathematical Functions (Dover, New York, 1965), p. 486.

13. Ref. 12, p. 360 .

14. B. A. Auld, Acoustic Fields and Waves in Solids (Wiley, New York, 1973).

15. A. Erdélyi, W. Magnus, F. Oberhettinger, and F. G. Tricomi, Tables of Integral Transforms (McGraw-Hill, New York, 1954), p. 277.

16. A. C. Tam and C. K. N. Patel, Appl. Opt. 18, 3348 (1979).

17. Y. Kohanzadeh, J. R. Whinnery, and M. M. Carroll, J. Acoust. Soc. Am. 57, 67 (1975).

18. C. P. Germano, J. Acoust. Soc. Am. 34, 1139 (1962).

19. R. A. Langevin, J. Acoust. Soc. Am. 26, 421 (1954). 